\title{
HIGHLIGHTS
}

RHEUMATOID ARTHRITIS

\section{Anti-TNF therapy for RA hinders function and survival of dendritic cells}

New research shows that blockade of tumor necrosis factor (TNF) leads to impaired dendritic cell survival and function, and provides insight into why TNF antagonists are effective treatments for rheumatoid arthritis (RA).

TNF is a well-known modulator of synovial inflammation, and anti-TNF therapy is commonly used to treat patients with RA. "Because TNF plays a central role in dendritic cell biology and dendritic cells are involved in rheumatoid arthritis pathogenesis, we decided to investigate how anti-TNF therapeutics modulate dendritic cell function," says senior author Catharien Hilkens from Newcastle University, UK.

Hilkens and colleagues first used in vitro studies to show that blockade of TNF increased apotosis of dendritic cells activated with lipopolysaccharide. Moreover, they found that treatment with TNF antagonists prevented the full maturation of the activated dendritic cells (with marked reductions in CD80, CD86 and CD83 expression). These "semi-mature" dendritic cells secreted notable levels of interleukin (IL)-10 and had a reduced immunostimulatory effect on T cells-in fact, they skewed the T-cell response to a more anti-inflammatory cytokine profile. The researchers observed that $\mathrm{T}$ cells primed with anti-TNF-treated dendritic cells secreted substantially higher levels of IL-10, IL-4 and IL-17 and, conversely, lower levels of interferon- $\gamma$ than $\mathrm{T}$ cells primed with fully matured dendritic cells.

Next, the investigators adopted an ex vivo approach to investigate whether antiTNF therapy influenced the function of dendritic cells from patients with RA. They generated monocyte-derived dendritic cells from patients before and after anti-TNF therapy. Again, they found that treatment with TNF antagonists influenced how dendritic cells matured and reduced their immunostimulation of T cells.

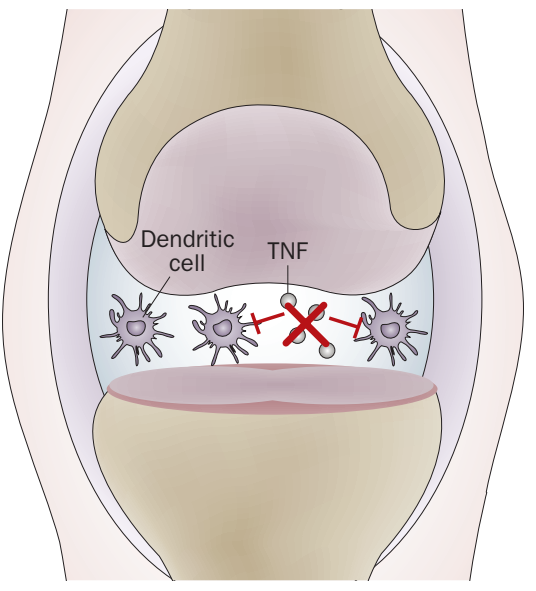

"Our findings suggest an additional mechanism by which anti-TNF therapy enhances immunoregulation," adds Hilkens, who now plans to study the in vivo outcomes of anti-TNF therapy on dendritic cells in patients with RA.

\section{Katrina Ray}

Original article Baldwin, H. M. et al. Tumour necrosis facto alpha blockade impairs dendritic cell survival and function in rheumatoid arthritis. Ann. Rheum. Dis. 69, 1200-1207 (2010) 